\title{
Degree Correlation Analysis Method of Mixed Networks*
}

\author{
Xianmin Geng ${ }^{1}$, Ping Qiu ${ }^{1}$, Shengli Zhou ${ }^{1,2}$, Zhiyuan Yao $^{2}$, and Zhijun Sun ${ }^{2}$ \\ ${ }^{1}$ College of Science Nanjing University of Aeronautics and Astronautics, Nanjing, China \\ ${ }^{2}$ State Key Laboratory of Mechanics and Control of Mechanical Structures, Nanjing University of Aeronautics and Astronautics, \\ Nanjing, China \\ zslmath@sohu.com, zslmnn@163.com
}

\begin{abstract}
In order to characterize the law of the degree correlations in the evolution of complex networks and explore the mechanism responsible for degree correlations, we investigate the degree correlations in mixed networks. We study the two-node degree correlations in networks with preferential attachment and intrinsic attachment. Using the dynamic system's rate equation approach under the assumption of continuous degree, we give the analytical result for two-node degree correlations, expressed by average nearest-neighbor degree (ANND). Analytical result shows that ANND decreases with respect to degree, which means that networks we studied are disassortative mixing. We also get the degree correlations of Barab $\phi s i-A l b e r t$ model, which is in the line with the existed result. Finally, the analytical result is in agreement with the numerical simulation.

Index Terms - Intrinsic link, Scale-free property, Degree correlation, Disassortative mixing.
\end{abstract}

\section{I . Introduction}

In the recent years, with the studies of many complex realword networks of diverse nature, e.g., social networks, biological networks, electronic communication networks, etc., the complex network has become the topic of academic interest $[1,2,3]$. The pioneering article of $\mathrm{R}$. Albert and Barab $\phi s i$ started the advanced research of the scale-free network [4,5]. In the evolution of the scale-free network, the evolution of next time step depends on the degree of each node on the current time step. Therefore, degree correlations play an important role in the characterization of the network topology. The two-node degree correlations reflect the fact that the degrees of the two ends of one randomly chosen link in the network are not independent. When degree correlations are positive, nodes with a large degree tend to connect to each other, and the network is said to be assortative. Conversely, when degree correlations are negative, nodes with large degree tend to connect to node with small degree instead of linking to each other, and the network is said to be disassortative.[6,7].

R. Pastor-Satorras and A. Vazquez measured the two-node degree correlations by means of quantity, called average nearest-neighbour degree (ANND), which is a function of degree [8]. The ANND is defined as

$$
\bar{k}_{n n}(k)=\sum_{k^{\prime}} k^{\prime} P\left(k^{\prime} \mid k\right)
$$

where $\bar{k}_{n n}(k)$ is the average nearest-neighbour degree nodes with degree $k, P\left(k^{\prime} \mid k\right)$ is the conditional probability that a node with degree $k$ connects to a node with degree $k^{\prime}$. If there no two-node degree correlations, $\bar{k}_{n n}(k)$ is independent of $k$. When $\bar{k}_{n n}(k)$ decreases (increases) as $k$ increases, the network shows disassortative mixing (or assortative mixing).

Zhongzhi Zhang and Shuigeng Zhou proved that in Random Apollonian Network the two-node correlation is independent of the degree [9]. Alain Barrat and R. PastorSatorras proved that the degree correlations in Linear Preferential Attachment models depend on the parameter $a(-m<a<\infty)$ of the preferential probability $\Pi_{s}(k, t)=\frac{k_{s}(t)+a}{(2 m+a) t}$,

where $\Pi_{s}(k, t)$ denotes the probability that a new node links to an existed node $s$ preferentially at time $t$, and $k_{s}(t)$ denotes the degree of node $s$ at time $t$ [10]. Josep L. Garcia-Domingo and David Juher investigated the degree correlations in growing networks with deletion of node [11]. Xianmin Geng and Ying Wang considered the degree correlations in citation networks model with aging and found that the citation network is assortative mixing [12].

In this paper, we study the two-node degree correlations in networks with preferential attachment and intrinsic attachment. Intrinsic link means when we add a new link to the network, the connection of some two kinds of nodes is inevitable. The inevitability is determined by the intrinsic features of two individuals in particular network [13]. These features determine that the appearance of some links is no longer in line with preferential attachment mechanism, but with the certainty. If we regard the whole network as a uniform network, the status of each node is equal and the intrinsic attachment mechanism shows randomicity on the connection probability.

Many real systems reflect the characteristics of intrinsic link. For example, in the World Wide Web (WWW), when a new webpage of a website is added, it must have a hyperlink with the homepage of the website. In addition, in the social network, relationships between parents and children, business relationships between the branch companies or business ralationships between the branch companies and the parent company, etc., are all containing the characteristics of intrinsic links.

The model we studied in this paper is formed by following four mechanisms: the addition of new nodes with preferential

\footnotetext{
* This work is supported by NNSF (grant No.11171215), NNSF (grant No.51275229) in China, and the National Basic Research Program (973 Program) (2011CB707602).
} 
attachment, the addition of new nodes with intrinsic attachment, the addition of new links with preferential attachment and the addition of new links with intrinsic attachment. And each time step, we perform one of the above four operations with probability. The degree distribution has been discussed in detail in ref. [13]. On the basis of this, using the rate equation approach, this paper obtains the upper bound of ANND of nodes with degree $k$ at time $t$. Further, from the estimation of $\bar{k}_{n n}(k, t)$, we found that for large $t$, node with large degree tends to connect to nodes with small degree, that is mixed networks are disassortative mixing.

The rest of this paper is organised as follows: In Sec. 2, we introduce our model and give some existed results. In Sec. 3, using the rate equation approach, we get the upper bound of ANND and then we perform numerical simulation. Finally, the last section summarizes the paper.

\section{II . Model of mixed networks}

In this section, we will review the model of mixed networks in ref. [13] and give some results about the model obtained in ref. [13], which are necessary for the research of degree correlations.

The model dynamics from a connected configuration consisted of $m_{0}$ nodes. We denote by $N(t)$ the size of the system at time $t$, and by $k_{i}(t)$, the degree of the node $i$ at time step $t$. At each time step, we perform one of the following four operations:

1) The addition of a new node with preferential attachment: a new node $s$ with $m\left(m \leq m_{0}\right)$ new links to the network, each new link is connected with an existed node $i$ with the preferential probability $\Pi_{s \rightarrow i}=\frac{k_{i}(t)}{\sum_{j} k_{j}(t)}$. We perform this operation with the probability $p_{1}$.

2) The addition of a new node with intrinsic attachment: a new node $s$ with $m\left(m \leq m_{0}\right)$ intrinsic links to the network, each new link is connected with an existed node $i$ with the preferential probability $\Pi_{s \rightarrow i}=\frac{1}{N(t)}$. We perform this operation with the probability $p_{2}$.

3) The addition of $n$ new node with preferential attachment: select a node $j$ as the starting point of the new link randomly, while a node $i$ is selected as the other end of the new link with the preferential probability $\frac{k_{i}(t)}{\sum_{\alpha} k_{\alpha}(t)}$. This operation is repeated $n$ times. We perform this operation with the probability $q_{1}$.

4) The addition of $n$ new intrinsic links: the two ends of a new intrinsic link are selected with probability $\frac{1}{N(t)}$. This operation is repeated $n$ times.

We perform this operation with probability $q_{2}$, where $q_{2}=1-p_{1}-p_{2}-q_{1}$. We assume $p_{1}, p_{2}, q_{1}$ and $q_{2}>0$. The node $i$ is added to the system at time $t_{i}$, if $t$ is large enough, we can get $N(t)=m_{0}+\left(p_{1}+p_{2}\right) t \approx p t, k_{i}\left(t_{i}\right)=m, \sum_{\alpha} k_{\alpha}(t)=a t$.

Where $p=p_{1}+p_{2}, a=2\left(p_{1} m+p_{2} m+q_{1} n+q_{2} n\right)$.

By ref. [13], we know the model can self-organize into a scalefree network under the assumption: $p_{1}, p_{2}, q_{1}$ and $q_{2}>0$. The expression of the degree of all nodes at time $t$ is also obtained in ref. [13],

$$
k_{i}(t)=(m+A)\left(\frac{t}{t_{i}}\right)^{\beta}-A
$$

$$
\begin{aligned}
\text { where } \beta & =\frac{p_{1} m+q_{1} n}{2\left(p_{1} m+p_{2} m+q_{1} n+q_{2} n\right)}, \\
A & =\frac{2\left(p_{2} m+q_{1} n+2 q_{2} n\right)}{\left(p_{1}+p_{2}\right)} \cdot \frac{\left(p_{1} m+p_{2} m+q_{1} n+q_{2} n\right)}{\left(p_{1} m+q_{1} n\right)}
\end{aligned}
$$

\section{III . Analysis of degree correlations}

In this section, we will research the two-node degree correlations, and we want to get the analytical expression of the ANND.

We can analytically compute the function value of $\bar{k}_{n n}(k)$ for the model of mixed networks. Let $R_{i}(t)$ denotes the sum of degrees of the neighbours of node $i$ at time $t$. That is

$$
R_{i}(t)=\sum_{j \in \Omega_{i}} k_{j}(t)
$$

where $\Omega_{i}$ is the set of neighbours of node $i$. The average nearest-neighbour degree of node $i$ at time $t, \bar{k}_{n n}(i, t)$, is then given by $\bar{k}_{n n}(i, t)=R_{i}(t) / k_{i}(t)$. Since node $i$ is arbitrary chosen and $k_{i}(t)=(m+A)\left(\frac{t}{t_{i}}\right)^{\beta}-A$, we can get $t_{i}$ as a function of $k$ and $t$ when $k_{i}(t)=k$, namely, $t_{i}=t\left(\frac{k+A}{m+A}\right)^{-\frac{1}{\beta}}$. Plugging $t_{i}=t\left(\frac{k+A}{m+A}\right)^{-\frac{1}{\beta}}$ into $\bar{k}_{n n}(i, t)=R_{i}(t) / k_{i}(t)$, we obtain the average nearestneighbour degree of nodes with degree $k$ at time $t$, namely, $\bar{k}_{n n}(k, t)$.

\section{A. Rate equation for $R_{i}(t)$}

According to the continuum theory, we assume that $k_{i}(t)$ changes continuously. During the growth of the network, we can get rate equation for $R_{i}(t)$ by the following four cases:

1) Performing operation 1) at time $t$

$$
\left(\frac{d R_{i}(t)}{d t}\right)_{1}=p_{1}\left(m \cdot C_{m}^{1} \frac{k_{i}(t)}{\sum_{\alpha} k_{\alpha}(t)}+1 \cdot \sum_{j \in \Omega_{i}} C_{m}^{1} \frac{k_{j}(t)}{\sum_{\alpha} k_{\alpha}(t)}\right),
$$

where $C_{m}^{1} \frac{k_{i}(t)}{\sum_{\alpha} k_{\alpha}(t)}$ is the preferential probability that a new node $s$ connects to any existed node. Then when the new node links to $i, R_{i}(t)$ increases by $m$. And if one new link randomly connects to $j\left(j \in \Omega_{i}\right), R_{i}(t)$ increases by 1 . Considering all neighbours of $i$, we can get the above expression. 
2)

\section{Performing operation 2) at time $t$}

$$
\left(\frac{d R_{i}(t)}{d t}\right)_{2}=p_{2}\left(m \cdot C_{m}^{1} \frac{1}{N(t)}+1 \cdot \sum_{j \in \Omega_{i}} C_{m}^{1} \frac{1}{N(t)}\right),
$$

where $C_{m}^{1} \frac{1}{N(t)}$ is the probability that one link of the node randomly link to any existed node. Then when the new node links to $i, R_{i}(t)$ increases by $m$. And if one new link randomly connects to $j\left(j \in \Omega_{i}\right), R_{i}(t)$ increases by 1 .

\section{3) Performing operation 3) at time $t$}

Above all, we will give the probability that a new link connects to any existed node preferentially at time $t$. In this model, at time step $t$, a new link connects to any existed node preferentially with the

probability

$$
\sum_{k=m}^{N(t)-1} \frac{k}{\sum_{i} k_{i}(t)} \mu_{k}(t)=\frac{1}{\sum_{i} k_{i}(t)} \sum_{k=m}^{N(t)-1} k \mu_{k}(t)=\frac{1}{\sum_{i} k_{i}(t)} \frac{\sum_{i} k_{i}(t)}{N(t)-1} \approx \frac{1}{p t},
$$

where $\mu_{k}(t)$ is the proportion of the amount of nodes with degree $k$ in the network at time $t$. On the basis of this, we consider the changes of $R_{i}(t)$ by the following three cases:

(i) Node $i$ and $l$ are selected as the two ends of new link, where node $l$ is neither $i$ nor the neighbour of $i$. Then $R_{i}(t)$ increases by $k_{l}(t-1)+1$,

$$
\left(\frac{d R_{i}(t)}{d t}\right)_{(i)}=\sum_{k=m}^{N(t-1)-1} q_{1} n(k+1)\left(\frac{1}{N(t)} \sum_{l \in \Omega_{i}^{\prime}} \frac{1}{p t}+\frac{1}{p t} \sum_{l \in \Omega_{i}} \frac{1}{N(t)}\right) \mu_{k}(t-1),
$$

where $\Omega_{i}^{\prime}$ is the set of node which is neither $i$ nor the neighbour of $i \cdot \frac{1}{N(t)} \sum_{l \in \Omega_{i}} \frac{1}{p t}$ is the probability that $i$ is selected as the starting point of the new link randomly and $l\left(l \in \Omega_{i}^{\prime}\right)$ is selected as the other end of new link preferentially, $\frac{1}{p t} \sum_{l \in \Omega_{i}^{i}} \frac{1}{N(t)}$ is the probability that $l\left(l \in \Omega_{i}^{\prime}\right)$ is selected as the starting point of the new link randomly and $i$ is selected as the other end of new link preferentially

(ii) Two neighbours of $i$ are selected as the two ends of new link, then $R_{i}(t)$ increases by 2 . So

$$
\left(\frac{d R_{i}(t)}{d t}\right)_{(i i)}=2 q_{1} n \sum_{e \in \Omega_{i}} \frac{1}{N(t)} \sum_{f \in \Omega_{i}, f \neq e} \frac{1}{p t} .
$$

(iii) Node $h$ and $l$ are selected as the two ends of new link, where $h \in \Omega_{i}, l \in \Omega_{i}^{\prime}$. Then $R_{i}(t)$ increases by 1 ,

$$
\left(\frac{d R_{i}(t)}{d t}\right)_{(i i i)}=q_{1} n\left(\sum_{h \in \Omega_{i}} \frac{1}{N(t)} \sum_{l \in \Omega_{i}^{i}} \frac{1}{p t}+\sum_{l \in \Omega_{i}} \frac{1}{N(t)} \sum_{h \in \Omega_{i}} \frac{1}{p t}\right) .
$$

where $\sum_{h \in \Omega_{i}} \frac{1}{N(t)} \sum_{l \in \Omega_{i}} \frac{1}{p t}$ is the probability that $h\left(h \in \Omega_{i}\right)$ is selected as the starting point of the new link randomly and $l\left(l \in \Omega_{i}^{\prime}\right)$ is selected as the other end of new link preferentially, $\sum_{l \in \Omega_{i}} \frac{1}{N(t)} \sum_{h \in \Omega_{\Omega}} \frac{1}{p t}$ is the probability that $l\left(l \in \Omega_{i}^{\prime}\right)$ is selected as the starting point of the new link randomly and $h\left(h \in \Omega_{i}\right)$ is selected as the other end of new link preferentially.

Hence, performing operation 3) at time $t$, we get

$$
\begin{aligned}
& \left(\frac{d R_{i}(t)}{d t}\right)_{z}-\left(\frac{d R_{2}(t)}{d t}\right)_{(t)}+\left(\frac{d R_{2}(t)}{d t}\right)_{(t)}+\left(\frac{d R_{l}(t)}{d t}\right)_{(m)}
\end{aligned}
$$

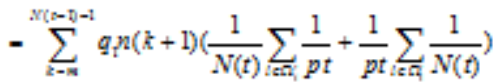

$$
\begin{aligned}
& \cdot \mu_{2}(t-1)+2 q_{1} n \sum_{t \in \Omega} \frac{1}{N(t)} \sum_{f \in a_{, f t \alpha}} \frac{1}{p t}
\end{aligned}
$$

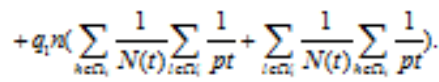

\section{4) Performing operation 4) at time $t$}

(i) Node $i$ and $l\left(l \in \Omega_{i}^{\prime}\right)$ are selected as the two ends of new link. Then $R_{i}(t)$ increases by $k_{l}(t-1)+1$,

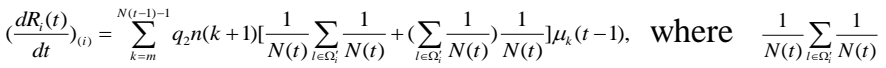

is the probability that $i$ is selected as the starting point and $l\left(l \in \Omega_{i}^{\prime}\right)$ is selected as the other end of new link preferentially, $\left(\sum_{l \in \Omega_{i}^{\prime}} \frac{1}{N(t)}\right) \frac{1}{N(t)}$ is the probability that $l\left(l \in \Omega_{i}^{\prime}\right)$ is selected as the starting point and $i$ is selected as the other end of new link randomly.

(ii) Two neighbours of $i$ are selected as the two ends of new link, then $R_{i}(t)$ increases by 2 . So

$$
\left(\frac{d R_{i}(t)}{d t}\right)_{(i i)}=2 q_{2} n \sum_{e \in \Omega_{i}} \frac{1}{N(t)}\left(\sum_{f \in \Omega_{i}, f \neq e} \frac{1}{N(t)}\right) .
$$

(iii) Node $h$ and $l$ are selected as the two ends of new link, where $h \in \Omega_{i}, l \in \Omega_{i}^{\prime}$. Then $R_{i}(t)$ increases by 1 ,

$$
\left(\frac{d R_{i}(t)}{d t}\right)_{(i i i)}=q_{2} n\left(\sum_{h \in \Omega_{i}} \frac{1}{N(t)} \sum_{l \in \Omega_{i}} \frac{1}{N(t)}+\sum_{l \in \Omega_{i}^{i}} \frac{1}{N(t)} \sum_{h \in \Omega_{i}} \frac{1}{N(t)}\right) .
$$

where $\sum_{h \in \Omega_{i}} \frac{1}{N(t)} \sum_{l \in \Omega_{i}} \frac{1}{N(t)}$ is the probability that $h\left(h \in \Omega_{i}\right)$ is selected as the starting point of the new link randomly and $l\left(l \in \Omega_{i}^{\prime}\right)$ is selected as the other end of new link randomly, $\left.\sum_{l \in \Omega_{i}} \frac{1}{N(t)} \sum_{h \in \Omega_{i}} \frac{1}{N(t)}\right)$ is the probability that $l\left(l \in \Omega_{i}^{\prime}\right)$ is selected as the starting point of the new link randomly and $h\left(h \in \Omega_{i}\right)$ is selected as the other end of new link randomly.

Hence, performing operation 4) at time $t$, we get

$$
\begin{aligned}
& \left(\frac{d R_{i}(t)}{d t}\right)_{4}=\left(\frac{d R_{i}(t)}{d t}\right)_{(i)}+\left(\frac{d R_{i}(t)}{d t}\right)_{(i i)}+\left(\frac{d R_{i}(t)}{d t}\right)_{(i i i)} \\
& =\sum_{k=m}^{N(t-1)-1} q_{2} n(k+1)\left[\frac{1}{N(t)} \sum_{l \in \Omega_{i}} \frac{1}{N(t)}+\left(\sum_{l \in \Omega_{i}} \frac{1}{N(t)}\right) \cdot \frac{1}{N(t)}\right] \mu_{k}(t-1) \\
& +2 q_{1} n \sum_{e \in \Omega_{i}} \frac{1}{N(t)} \cdot\left(\sum_{f \in \Omega_{i}, f \neq e} \frac{1}{N(t)}\right) \\
& +q_{2} n\left(\sum_{h \in \Omega_{i}} \frac{1}{N(t)} \cdot \sum_{l \in \Omega_{i}^{i}} \frac{1}{N(t)}+\sum_{l \in \Omega_{i}} \frac{1}{N(t)} \sum_{h \in \Omega_{i}} \frac{1}{N(t)}\right) .
\end{aligned}
$$


So we can obtain the rate equation for $R_{i}(t)$ :

$$
\frac{d R_{i}(t)}{d t}=\left(\frac{d R_{i}(t)}{d t}\right)_{1}+\left(\frac{d R_{i}(t)}{d t}\right)_{2}+\left(\frac{d R_{i}(t)}{d t}\right)_{3}+\left(\frac{d R_{i}(t)}{d t}\right)_{4} .
$$

We observe that at time $t_{i}$, the new node $i$ is connected to an existed node $j$ with degree $k_{j}\left(t_{i}\right)$ with probability $p_{1} C_{m}^{1} \sum_{\alpha}^{\frac{k_{j}}{t_{\alpha}} k_{\alpha}\left(t_{i}\right)}+p_{2} C_{m}^{\frac{1}{n}} \frac{1}{N\left(t_{i}\right)}$, and then the degree of $j$ increases by 1 in the process. When we focus on all the nodes that entered the system before node $i$, we can obtain the boundary condition for (8) by

$$
R_{i}\left(t_{i}\right)=\sum_{j=1}^{N\left(t_{i}\right)-1}\left(p_{1} C_{m}^{1} \frac{k_{j}\left(t_{i}\right)}{\sum_{\alpha} k_{\alpha}\left(t_{i}\right)}+p_{2} C_{m}^{1} \frac{1}{N\left(t_{i}\right)}\right)\left(k_{j}\left(t_{i}\right)+1\right) .
$$

\section{B. Calculation of $R_{i}(t)$}

Plugging $R_{i}(t)=\sum_{j \in \Omega_{i}} k_{j}(t), \sum_{\alpha} k_{\alpha}(t)=a t, N(t) \approx p t$, and (4), (5),

(6), (7) into (8), and noting that $\sum_{k=m}^{N(-1-1)-1}(k+1) \mu_{k}(t-1)=\frac{a+p}{p}$,

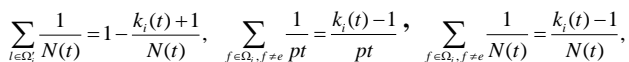

we have

$$
\begin{aligned}
\frac{d R_{i}(t)}{d t}= & \frac{m p_{1}}{a} \frac{R_{i}(t)}{t}+\left(\frac{m^{2} p_{1}}{a}+\frac{m p_{2}}{p}\right) \frac{k_{i}(t)}{t} \\
& +\frac{m^{2} p_{2}}{a} \frac{1}{t}+2\left(q_{1}+q_{2}\right) n \frac{a+p}{p^{2} t}\left(1-\frac{k_{i}(t)+1}{p t}\right) \\
& +2\left(q_{1}+q_{2}\right) n \frac{k_{i}(t)}{p t} \frac{k_{i}(t)-1}{p t} \\
& +2\left(q_{1}+q_{2}\right) n \frac{k_{i}(t)}{p t}\left(1-\frac{k_{i}(t)+1}{p t}\right) .
\end{aligned}
$$

By $k_{i}(t)=(m+A)\left(\frac{t}{t_{i}}\right)^{\beta}-A$ and $A>0$, we know $k_{i}(t) \leq(m+A)\left(\frac{t}{t_{i}}\right)^{\beta}$, $k_{i}(t)\left(k_{i}(t)-1\right) \leq(m+A)\left(\frac{t}{t_{i}}\right)^{2 \beta}$. Since $1-\frac{k_{i}(t)+1}{p t} \leq 1,(10)$ becomes

$$
\begin{aligned}
\frac{d R_{i}(t)}{d t} \leq & \frac{m p_{1}}{a} \frac{R_{i}(t)}{t}+\left(\frac{m^{2} p_{1}}{a}+\frac{m p_{2}}{p}+c\right)(m+A)\left(\frac{t}{t_{i}}\right)^{\beta} \frac{1}{t} \\
& +\left[\frac{m^{2} p_{2}}{p}+\frac{2 n\left(q_{1}+q_{2}\right)(a+p)}{p^{2}}\right] \frac{1}{t} \\
& +\frac{2 n\left(q_{1}+q_{2}\right)}{p^{2}}(m+A)^{2}\left(\frac{t}{t_{i}}\right)^{2 \beta} \frac{1}{t^{2}} .
\end{aligned}
$$

Namely,

$$
\frac{d R_{i}(t)}{d t} \leq c \frac{R_{i}(t)}{t}+f(t),
$$

where $c=\frac{m p_{1}}{a}$ and

$$
\begin{aligned}
f(t)= & \left(\frac{m^{2} p_{1}}{a}+\frac{m p_{2}}{p}+\frac{2 n\left(q_{1}+q_{2}\right)}{p}\right)(m+A)\left(\frac{t}{t_{i}}\right)^{\beta} \frac{1}{t} \\
& +\left[\frac{m^{2} p_{2}}{p}+\frac{2 n\left(q_{1}+q_{2}\right)(a+p)}{p^{2}}\right] \frac{1}{t} \\
& +\frac{2 n\left(q_{1}+q_{2}\right)}{p^{2}}(m+A)^{2}\left(\frac{t}{t_{i}}\right)^{2 \beta} \frac{1}{t^{2}} .
\end{aligned}
$$

Multiplying $t^{-c}$ to both sides of (12), we obtain

$$
t^{-c} \frac{d R_{i}(t)}{d t}-c t^{-(c+1)} R_{i}(t) \leq t^{-c} f(t) .
$$

Namely,

$$
\frac{d\left(t^{-c} R_{i}(t)\right)}{d t} \leq t^{-c} f(t)
$$

Taking integration for the trajectory of $t$, we obtain

$$
\frac{R_{i}(t)}{t^{c}}-\frac{R_{i}\left(t_{i}\right)}{t_{i}^{c}} \leq \int_{t_{i}}^{t} \frac{f(\tau)}{\tau^{c}} d \tau
$$

Namely,

$$
R_{i}(t) \leq t^{c}\left(\frac{R_{i}\left(t_{i}\right)}{t_{i}^{c}}+\int_{t_{i}}^{t} \frac{f(\tau)}{\tau^{c}} d \tau\right),
$$

where

$$
\begin{aligned}
\int_{t_{i}}^{t} \frac{f(\tau)}{\tau^{c}} d \tau & =\frac{m+A}{\beta-c}\left(\frac{m^{2} p_{1}}{a}+\frac{m p_{2}+2 n\left(q_{1}+q_{2}\right)}{p}\right)\left(t^{\beta-c}-t_{i}^{\beta-c}\right) t_{i}^{-\beta} \\
& +\left(\frac{m^{2} p_{2}}{-c p}+\frac{2 n\left(q_{1}+q_{2}\right)(a+p)}{-c p^{2}}\right)\left(t^{-c}-t_{i}^{-c}\right) \\
& +\frac{2 n\left(q_{1}+q_{2}\right)(m+A)^{2}}{(2 \beta-c-1) p^{2}}\left(t^{2 \beta-c-1}-t_{i}^{2 \beta-c-1}\right) t_{i}^{-2 \beta} .
\end{aligned}
$$

Plugging $\sum_{\alpha} k_{\alpha}\left(t_{i}\right)=a t_{i}$ and $N\left(t_{i}\right) \approx p t_{i}$ into (9), and noting that $\frac{a t_{i}-m}{a t_{i}} \leq 1, \quad \frac{a t_{i}-m}{p t_{i}} \leq \frac{a}{p}, \quad \frac{p t_{i}-1}{p t_{i}} \leq 1, \quad k^{2}{ }_{j}\left(t_{i}\right) \leq(m+A)^{2}\left(\frac{t_{i}}{t_{j}}\right)^{2 \beta}$, $t_{j} \geq 1\left(j=1,2, \cdots, p t_{i}-1\right)$, we have

$$
\begin{aligned}
R_{i}\left(t_{i}\right) & =\sum_{j=1}^{p t_{i}-1} m p_{1} \frac{k_{j}^{2}\left(t_{i}\right)}{a}+m p_{1} \frac{a t_{i}-m}{a t_{i}}+m p_{21} \frac{a t_{i}-m}{p t_{i}}+m p_{2} \frac{p t_{i}-m}{p t_{i}} \\
& \leq \sum_{j=1}^{p_{i}-1} \frac{m p_{1}}{a}(m+A)^{2} t_{j}^{-2 \beta} t_{i}^{2 \beta-1}+m p_{1}+m p_{2}\left(1+\frac{a}{p}\right) \\
& \leq\left(p t_{i}-1\right) \frac{m p_{1}}{a}(m+A)^{2} t_{i}^{2 \beta-1}+m p_{1}+m p_{2}\left(1+\frac{a}{p}\right) \\
& \leq \frac{m p p_{1}}{a}(m+A)^{2} t_{i}^{2 \beta}+m\left[p_{1}+p_{2}\left(1+\frac{a}{p}\right)\right] .
\end{aligned}
$$

Plugging (18), (19) into (17), we have

$$
\begin{aligned}
R_{i}\left(t_{i}\right) \leq & m\left[p_{1}+p_{2}\left(1+\frac{a}{p}\right)\right]\left(\frac{t}{t_{i}}\right)^{c}+\frac{m p p_{1}}{a}(m+A)^{2} t_{i}^{2 \beta}\left(\frac{t}{t_{i}}\right)^{c} \\
& +\frac{m+A}{\beta-c}\left(\frac{m^{2} p_{1}}{a}+\frac{m p_{2}+2 n\left(q_{1}+q_{2}\right)}{p}\right)\left[\left(\frac{t}{t_{i}}\right)^{\beta}-\left(\frac{t}{t_{i}}\right)^{c}\right] \\
& +\left(\frac{m^{2} p_{2}}{c p}+\frac{2 n\left(q_{1}+q_{2}\right)(a+p)}{c p^{2}}\right)\left[\left(\frac{t}{t_{i}}\right)^{c}-1\right] \\
& +\frac{2 n\left(q_{1}+q_{2}\right)(m+A)^{2}}{(1+c-2 \beta) p^{2}}\left[\left(\frac{t}{t_{i}}\right)^{1+c}-\left(\frac{t}{t_{i}}\right)^{2 \beta}\right] t^{-1} .
\end{aligned}
$$

Since $t>t_{i}, 0<c<\beta \leq \frac{1}{2}$, we have $\left(\frac{t}{t_{i}}\right)^{c} \geq 1,\left(\frac{t}{t_{i}}\right)^{2 \beta} \geq 1$. Noting that $\quad a=2\left(p_{1} m+p_{2} m+q_{1} n+q_{2} n\right) \quad, \quad \beta=\frac{p_{1} m+q_{1} n}{a} \quad, \quad c=\frac{m p_{1}}{a}$, $A=\frac{2\left(p_{2} m+q_{1} n+2 q_{2} n\right)}{\left(p_{1}+p_{2}\right)} \cdot \frac{\left(p_{1} m+p_{2} m+q_{1} n+q_{2} n\right)}{\left(p_{1} m+q_{1} n\right)} \quad, \quad p=p_{1}+p_{2}$, 
$\frac{m+A}{\beta-c}\left(\frac{m^{2} p_{1}}{a}+\frac{m p_{2}+2 n\left(q_{1}+q_{2}\right)}{p}\right)>0$, and $\frac{2 n\left(q_{1}+q_{2}\right)(m+A)^{2}}{(1+c-2 \beta) p^{2}}>0$ ， (20) becomes

$$
\begin{aligned}
R_{i}\left(t_{i}\right) \leq & m\left[p_{1}+p_{2}\left(1+\frac{a}{p}\right)\right]\left(\frac{t}{t_{i}}\right)^{c}+\frac{m p p_{1}}{a}(m+A)^{2} t_{i}^{2 \beta}\left(\frac{t}{t_{i}}\right)^{c} \\
& +\frac{m+A}{\beta-c}\left(\frac{m^{2} p_{1}}{a}+\frac{m p_{2}+2 n\left(q_{1}+q_{2}\right)}{p}\right)\left[\left(\frac{t}{t_{i}}\right)^{\beta}-1\right] \\
& +\left(\frac{m^{2} p_{2}}{c p}+\frac{2 n\left(q_{1}+q_{2}\right)(a+p)}{c p^{2}}\right)\left[\left(\frac{t}{t_{i}}\right)^{c}-1\right] \\
& +\frac{2 n\left(q_{1}+q_{2}\right)(m+A)^{2}}{(1+c-2 \beta) p^{2}}\left[\left(\frac{t}{t_{i}}\right)^{1+c}-1\right] t^{-1} .
\end{aligned}
$$

We denote the right side of (21) by $Q_{i}(t)$, then (21) reduces to

$$
R_{i}(t) \leq Q_{i}(t)
$$

\section{Calculation of $\bar{k}_{n n}(k, t)$}

By $\bar{k}_{n n}(i, t)=\frac{R_{i}(t)}{k_{i}(t)}, \quad k_{i}(t)=(m+A)\left(\frac{t}{t_{i}}\right)^{\beta}-A$, and $R_{i}(t) \leq Q_{i}(t)$, noting that $A>0$ we have

$$
\bar{k}_{n n}(i, t) \leq \frac{Q_{i}(t)}{k_{i}(t)} \leq \frac{Q_{i}(t)+A}{(m+A)\left(\frac{t}{t_{i}}\right)^{\beta}} .
$$

Plugging $Q_{i}(t)$ into (23), we obtain

$$
\begin{aligned}
\bar{k}_{n n}(i, t) \leq & m(m+A)^{-1}\left[p_{1}+p_{2}\left(1+\frac{a}{p}\right)\right]\left(\frac{t}{t_{i}}\right)^{c-\beta}+\frac{m p p_{1}}{a}(m+A) t_{i}^{2 \beta}\left(\frac{t}{t_{i}}\right)^{c-\beta} \\
& +\frac{1}{\beta-c}\left(\frac{m^{2} p_{1}}{a}+\frac{m p_{2}+2 n\left(q_{1}+q_{2}\right)}{p}\right)\left[1-\left(\frac{t}{t_{i}}\right)^{-\beta}\right] \\
& +\left(\frac{m^{2} p_{2}}{c p}+\frac{2 n\left(q_{1}+q_{2}\right)(a+p)}{c p^{2}}\right)(m+A)^{-1}\left(\frac{t}{t_{i}}\right)^{c-\beta} \\
& -\left(\frac{m^{2} p_{2}}{c p}+\frac{2 n\left(q_{1}+q_{2}\right)(a+p)}{c p^{2}}\right)(m+A)^{-1}\left(\frac{t}{t_{i}}\right)^{-\beta} \\
& +\frac{2 n\left(q_{1}+q_{2}\right)(m+A)}{(1+c-2 \beta) p^{2}}\left[\left(\frac{t}{t_{i}}\right)^{1+c}-1\right]\left(\frac{t}{t_{i}}\right)^{-\beta} t^{-1}+A(m+A)^{-1}\left(\frac{t}{t_{i}}\right)^{-\beta} .
\end{aligned}
$$

Plugging $t_{i}=t\left(\frac{k+A}{m+A}\right)^{-\frac{1}{\beta}}$ into (24), we obtain the upper bound of average nearest-neighbour degree of nodes with degree $k$ at time step $t$ :

$$
\begin{aligned}
\bar{k}_{n n}(k, t) \leq & m(m+A)^{-\frac{c}{\beta}}\left[p_{1}+p_{2}\left(1+\frac{a}{p}\right)\right](k+A)^{-1+\frac{c}{\beta}} \\
& +\frac{m p p_{1}}{a}(m+A)^{4-\frac{c}{\beta}}(k+A)^{-3+\frac{c}{\beta}} t_{i}^{2 \beta} \\
& +\frac{1}{\beta-c}\left(\frac{m^{2} p_{1}}{a}+\frac{m p_{2}+2 n\left(q_{1}+q_{2}\right)}{p}\right)\left[1-\left(\frac{k+A}{m+A}\right)^{-1}\right] \\
& +\left(\frac{m^{2} p_{2}}{c p}+\frac{2 n\left(q_{1}+q_{2}\right)(a+p)}{c p^{2}}\right)(m+A)^{-\frac{c}{\beta}}(k+A)^{-1+\frac{c}{\beta}} \\
& -\left(\frac{m^{2} p_{2}}{c p}+\frac{2 n\left(q_{1}+q_{2}\right)(a+p)}{c p^{2}}\right)(k+A)^{-1} \\
& +\frac{2 n\left(q_{1}+q_{2}\right)(m+A)}{(1+c-2 \beta) p^{2}}\left[\left(\frac{k+A}{m+A}\right)^{\frac{1+c}{\beta}-1}-\left(\frac{k+A}{m+A}\right)^{-1}\right] t^{-1} \\
& +A(k+A)^{-1}
\end{aligned}
$$

In order to investigate the changes of $\bar{k}_{n n}(k, t)$ with respect to $k$, we consider the following two inequalities:

$$
\begin{aligned}
& \left(-3+\frac{c}{\beta}\right) \frac{m p p_{1}}{a}(m+A)^{4-\frac{c}{\beta}}(k+A)^{-4+\frac{c}{\beta}} t^{2 \beta} \\
& +\frac{m+A}{\beta-c}\left(\frac{m^{2} p_{1}}{a}+\frac{m p_{2}+2 n\left(q_{1}+q_{2}\right)(a+p)}{p}\right)(k+A)^{-2} \\
& +\left(\frac{m^{2} p_{2}}{c p}+\frac{2 n\left(q_{1}+q_{2}\right)(a+p)}{c p^{2}}\right)(k+A)^{-2}<0,
\end{aligned}
$$

and

$$
\begin{aligned}
& m(m+A)^{-\frac{c}{\beta}}\left[p_{1}+p_{2}\left(1+\frac{a}{p}\right)\right]\left(-1+\frac{c}{\beta}\right)(k+A)^{-2+\frac{c}{\beta}} \\
& +\left(\frac{m^{2} p_{2}}{c p}+\frac{2 n\left(q_{1}+q_{2}\right)(a+p)}{c p^{2}}\right)\left(-1+\frac{c}{\beta}\right)(m+A)^{-\frac{c}{\beta}}(k+A)^{-2+\frac{c}{\beta}} \\
& +\frac{2 n\left(q_{1}+q_{2}\right)}{(1+c-2 \beta) p^{2}}\left(\frac{1+c}{\beta}-1\right)\left(\frac{k+A}{m+A}\right)^{\frac{1+c}{\beta}-1} t^{-1} \\
& +\frac{2 n\left(q_{1}+q_{2}\right)}{(1+c-2 \beta) p^{2}}\left(\frac{k+A}{m+A}\right)^{-2} t^{-1}-A(k+A)^{-2} \\
& \leq 0,
\end{aligned}
$$

where $_{0<c<\beta \leq \frac{1}{2}},-1+\frac{c}{\beta}<0,-3+\frac{c}{\beta}<0$. If (26) holds, we have

$$
t>\left(\frac{a \beta}{3 \beta-c} \frac{C_{1}(m+A)+D_{1}}{m p p_{1}}\right)^{\frac{1}{2 \beta}}(m+A)^{\frac{-4 \beta+c}{2 \beta^{2}}}(k+A)^{\frac{2 \beta-c}{2 \beta^{2}}}=T_{1} .
$$

If (27) holds, we have

$$
\begin{aligned}
t> & \frac{N(m+A)^{2-\frac{1+c}{\beta}}\left(-1+\frac{1+c}{\beta}\right)(k+A)^{\frac{1+c}{\beta}}}{M(m+A)^{-\frac{c}{\beta}}\left(1-\frac{c}{\beta}\right)(k+A)^{\frac{c}{\beta}}+A} \\
+ & \frac{N(m+A)^{2}}{M(m+A)^{-\frac{c}{\beta}}\left(1-\frac{c}{\beta}\right)(k+A)^{\frac{c}{\beta}}+A} \\
= & T_{2 .}
\end{aligned}
$$

Where

$$
\begin{aligned}
& C_{1}=\frac{m^{2} p_{1}}{a(\beta-c)}+\frac{m p_{2}+2 n\left(q_{1}+q_{2}\right)(a+p)}{p(\beta-c)}, D_{1}=\frac{m^{2} p_{2}}{c p}+\frac{2 n\left(q_{1}+q_{2}\right)(a+p)}{c p^{2}}, \\
& M=m p_{1}+\frac{m p_{2}(a+p)}{p}+\frac{m^{2} p_{2}}{c p}+\frac{2 n\left(q_{1}+q_{2}\right)(a+p)}{c p^{2}}, N=\frac{2 n\left(q_{1}+q_{2}\right)}{(1+c-2 \beta) p^{2}} .
\end{aligned}
$$

So in our model, when $t \geq \max \left\{T_{1}, T_{2}\right\}$, the average nearestneighbour degree of the nodes with degree $k$ increases as $k$ decreases. The result shows that mixed networks are disasortative mixing.

Specially, when $p_{1}=1, p_{2}=0, q_{1}=0, n=0, m>0$, by Ref. [13] we know $k_{i}(t)=\left(\frac{t}{t_{i}}\right)^{\beta}, \beta=\frac{1}{2}$. The model becomes Barab $\phi s i-$ Albert model, then $N(t)=t$ and (8) can be rewritten as

$$
\frac{d R_{i}(t)}{d t}=m^{2} \frac{k_{i}(t)}{\sum_{j} k_{j}(t)}+m \frac{k_{j}(t)}{\sum_{\alpha} k_{\alpha}(t)}=\frac{m^{2}}{2} t^{-\frac{1}{2}} t_{i}^{-\frac{1}{2}}+\frac{R_{i}(t)}{2 t} .
$$

Then we obtain 


$$
R_{i}(t)=\sqrt{\frac{t}{t_{i}}} R_{i}\left(t_{i}\right)+\frac{m^{2}}{2} \sqrt{\frac{t}{t_{i}}} \ln \frac{t}{t_{i}} .
$$

And $R_{i}\left(t_{i}\right)$ becomes

$$
R_{i}\left(t_{i}\right)=\sum_{j=1}^{i-1} C_{m}^{1} \frac{k_{j}\left(t_{i}\right)}{\sum_{\alpha} k_{\alpha}\left(t_{i}\right)}\left(k_{j}\left(t_{i}\right)+1\right) .
$$

Plugging $k_{i}(t)=m\left(\frac{t}{t_{i}}\right)^{\frac{1}{2}}$ into (30) results in

$$
R_{i}\left(t_{i}\right)=\frac{m^{2}}{2} \sum_{j=1}^{i-1} t_{j}^{-1}+m \frac{2 m t_{i}-m}{2 m t_{i}} .
$$

For large $t_{i}, \sum_{j=1}^{i-1} t_{j}^{-1} \approx \ln t_{i}$, we obtain

$$
R_{i}\left(t_{i}\right) \approx \frac{m^{2}}{2} \ln t_{i},\left(\text { for large } t_{i}\right)
$$

Plugging (32) into (29), we get

$$
R_{i}\left(t_{i}\right) \approx \frac{m^{2}}{2} \sqrt{\frac{t}{t_{i}}} \ln t .
$$

By the definition of $\bar{k}_{n n}(i, t)$, we have

$$
\bar{k}_{n n}(i, t)=\frac{R_{i}(t)}{k_{i}(t)} \approx \frac{m}{2} \ln t,
$$

And finally we get the ANND of node with degree $k$ at time step $t$

$$
\bar{k}_{n n}(k, t) \approx \frac{m}{2} \ln t .
$$

That is the two node correlation is independent of the degree and increases logarithmically with system size, which is in line with the result in ref. [10].

\section{IV . Numerical Simulation}

In order to check the analytical result obtained in this paper, we have performed extensive numerical simulation of the mixed network. Fig. 1 shows the comparison between the analytical result and the simulation result. From the figure we can see that analytical result is consistent with the simulation result, and our analytical result is the upper bound of the network's average nearest-neighbour degree which is determined by (25).

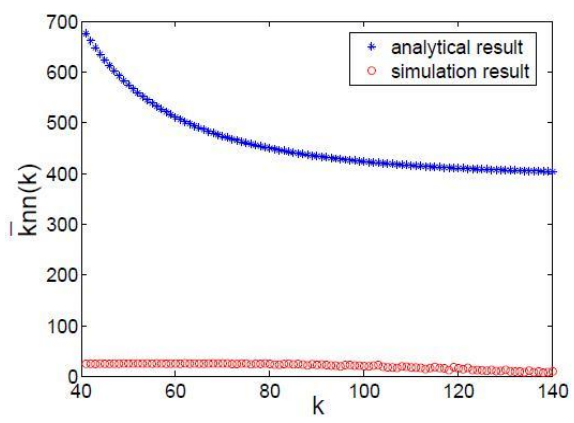

Figure 1 Average nearest-neighbor degree of the nodes.
Where the abscissa axis represents the degree of the nodes and the ordinate axis represents the average nearest-neighbor degree of the nodes. Symbols ' $O$ ' and '*' correspond to the simulation result and analytical result respectively. The simulation data are averaged over 50 networks generated by fixing $m_{0}=100, m=5, n=3, p_{1}=0.6, p_{2}=0.1, q_{1}=0.2$, $q_{1}=0.2, t=20000$.

\section{Conclusion}

In this paper, we presented an analytical study of the twonode degree correlations in mixed networks. Under the assumption of continuous degree, applying the rate equation approach, this paper studied the two-node degree correlations expressed by average nearest-neighbor degree(ANND). We have obtained the upper bound of ANND of nodes with degree $k$ at time step $t$, and found that for large $t$, the ANND decreases with respect to degree, that is the model we studied is disassortative. We also got the two-node degree correlations of t Barabasi-Albert model, which is in line with the existed result. Finally, we performed a numerical simulation and showed that the analytical result is in agreement with the numerical simulation.

Our paper mainly studied the two-node degree correlations of mixed networks. We calculate an upper bound of the average nearest-neighbor degree and then we get the negative degree correlations in our model. The derivation about the lower bound of the ANND is the problem that we will research further.

\section{References}

[1] M.E.J. Newman, "The structure and function of complex networks," SIAM Review, 45, pp. 167-256, 2003.

[2] M.E.J. Newman, S. H. Strogatz, D. J. Watts, "Random graphs with arbitrary degree distributions and their applications," Phys. Rev. E., 64, 026118, 2001.

[3] G. Lee, G. Il Kim, "Degree and wealth distribution in a network induced by wealth,” Physica A., 383, pp. 677-686, 2007.

[4] A. L. Barab'asi, R. Albert, "Emergence of Scaling in Random Networks," Science, 286, pp. 509-512, 1999.

[5] R. Albert, A. L. Barab'asi, "Statistical mechanics of complex networks," Reviews of Modern Physics. 74, pp. 47-97, 2002.

[6] M.E.J. Newman, “Assortative mixing in networks,” Phys. Rev. Lett., 89, pp. 2087012002.

[7] M.E.J. Newman, "Mixing patterns in networks," Phys. Rev. E. 67, 026126,2003

[8] R. Pastor-Satorras, A. V'azquez, A. Vespignani, "Dynamical and correlation properties of the Internet," Phys.Rev. Lett,. 87, 258701, 2001.

[9] Zhongzhi Zhang, Shuigeng Zhou, "Correlations in random Apollonian network," Physica A,. 380, pp. 621-628, 2007.

[10]A. Barrat, R. Pastor-Satorras, Phys. Rev. E., 71, 036127, 2005.

[11]Josep L. Garcia-Domingo, David Juher, Joan Saldana, "Degree correlations in growing networks with deletion of nodes," Phys. D., 237, pp. 640-651, 2008.

[12]Xianmin Geng, YingWang, "Degree correlations in citation networks model with aging," Europhysics Letters.88, 38002, 2009.

[13]Xianmin Geng, Guanghui Wen, Ying Wang, Jinxia Li, "EVOLVING MODEL OF SCALE-FREE NETWORKS WITH INTRINSIC LINKS," INT. J. MOD. PHYS. C., 19, pp. 1129-1144, 2008. 